\title{
The universal vector
}

\section{Abstract}

This paper takes Astrotheology mathematics and puts some of it in terms of linear algebra. All of physics can be described by two vectors. The dot product and cross product has important results. Here we describe the Gravity Equation. And we provide the coordinates of the universal and what they mean.

Keywords: linear algebra, astrotheology, gravity, mass, space
Volume I Issue 5 - 2018

\section{Paul TE Cusack}

Park Ave, Saint John, Canada

Correspondence: Paul TE Cusack, BScE, Dule 23 Park Ave, Saint John, NB E2J IR2, Canada, Tel 5066526350 Emailst-michael@hotmail.com

Received: August 0I, 2018 | Published:September 12, 2018

\section{Introduction}

In this paper, we provide calculations on Astrotheology from Linear Algebra. ${ }^{1}$ Einstein was wrong about no absolute space and time and these calculations show how. Gravity, Mass, Density, and the zero vectors are used in calculations that show that the universe can be modelled as a tupple. We begin with gravity.

Product

$$
\begin{aligned}
G_{0} & =|D| c \sqrt{c} \Omega \\
= & 4 \times 3 \times \sqrt{3} \times \pi \\
& =6.52
\end{aligned}
$$

Dot Product

$$
M=E \cdot t==|E| t \mid \cos \theta=0
$$

Sum

$$
\begin{aligned}
M_{0}= & |D|+c+\sqrt{c}+\Omega \\
= & 4+3+\sqrt{4}+\pi \\
& =118.72 \text { Mass in the periodic table of the }
\end{aligned}
$$

elements.

Cross Product

$$
\begin{gathered}
s=E \times t=|E| \mid t \sin \theta \\
G_{0} \cdot M=1 \\
x^{n} \int x=1 \\
x^{n} x^{2} / 2=1 \\
x^{2 n}=2
\end{gathered}
$$

Let $n=4$

$$
\begin{gathered}
x^{8}=2 \\
x=1.0905=1 / 917 \\
=\csc 66.6^{\circ}
\end{gathered}
$$

Dot Product $/$ Cross Product $=\cos \theta / \sin \theta=\cot \theta=\rho$

$$
\begin{gathered}
\theta=117.02 \\
=\text { Mass }=2.04 \text { rads } \\
x^{n} \int x=6.52=118 \\
n=4 \\
x^{2 n}=-/ 776 \\
x=126.7 \\
=\rho \\
Z=\rho[\cos \varphi+i \sin \varphi] \\
0.917 i=1.5 \\
1 /\left[x^{n} \int x\right] i=1.5 \\
i=1.5 x^{n} \int x \\
G 1+1 / 1=2=d M / d t \\
G=1 / t+1 / T \\
=1 / 1+1 / 251=1+1 /(1 / 4)=5 \\
x^{2}-x-1=5
\end{gathered}
$$

Roots 3, -2 = Eigenvalues.

$$
\begin{gathered}
F=G[M 1 M 2] / R^{2} \\
2(2)(1) /\left(1^{2}\right) \\
=4 \\
|D| \\
=M a \rho \\
=\text { Max } \\
=M a x^{n} \int x \\
=M a(1) \\
=M a \\
=F
\end{gathered}
$$


Golden mean

$$
\begin{gathered}
x^{2}-x-1=0 \\
\mathbb{C}: x+i y \\
(x+i y)^{2}-(x+i y)-1=0
\end{gathered}
$$

Reduces to the bilinear form

$$
\begin{aligned}
& x^{2}-y^{2}=1 \\
& x=a_{11} x^{\prime}+a_{12} y^{\prime} \mathrm{y} \\
&=a 21 x^{\prime}+a 22 y^{\prime}
\end{aligned}
$$

Rotation matrix

$$
\begin{aligned}
& \text { Let } \theta=\pi \\
& \pi=\Omega
\end{aligned}
$$

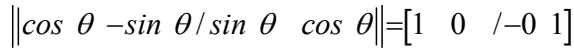

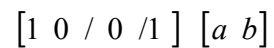

$$
\begin{aligned}
& {[a+0 b / 0 a-b]=\left[\begin{array}{ll}
a & -b
\end{array}\right]} \\
& {\left[\begin{array}{ll}
x & y
\end{array}\right][a 11-a 22==1} \\
& x a 11-a 22 y-1=0 \\
& a 11=x \\
& a 22=y \\
& x^{2}-y^{2}-1=0 \\
& a 11=A x \\
& a 22=C y \\
& A x^{2}+2 B x y+C y^{2}=D \\
& \text { (1) } x^{2}+2(-1) x y+1 y^{2}=0 \\
& x^{2}-2 x y+y^{2}=0 \\
& (1 / \sqrt{2})^{2}-2(1 / \sqrt{2})\left(1 /(\sqrt{2})+(1 / \sqrt{2})^{2}=0\right. \\
& 1 / 2-1+1 / 2=0 \\
& 0=0 \\
& x^{2}-2 x y+y^{2}=0 \\
& (x-y)(x-y)=0 \\
& x=y \\
& a=v \\
& P=F
\end{aligned}
$$

Figure 1

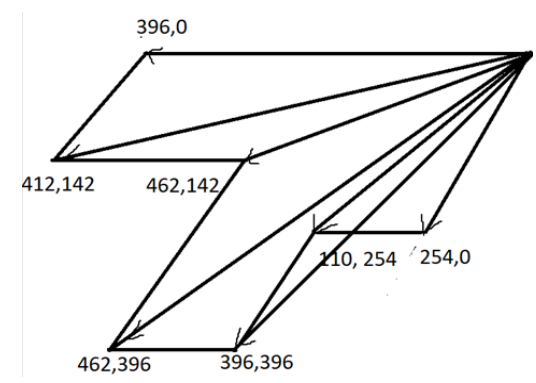

Figure I The universal vector space.
396,0

412,142

462,142

462,396

396,396

110,254

254,0

0,0

2492,1330

$[T, s]$

Multiple be Operator Matrix

$\left[\cos 60^{\circ}-\sin 60^{\circ}, \sin 60^{\circ}, \cos 60^{\circ}\right]$

$\left[\begin{array}{llll}1 / 2 & \sqrt{3} / 2, & \sqrt{3} / 2 & 1 / 2\end{array}\right]$

$|D|=[1 / 2 \times 1 / 2-\sqrt{3} / 2 \sqrt{3} / 2]$

[1/4-3/4]

$=[-1 / 2]$

Multiple by $[T, s]$

$$
\begin{gathered}
{\left[-1 / 2^{*} 251,4 / 3^{*}-1 / 2\right]} \\
{[-1 / 8,-2 / 3]}
\end{gathered}
$$

[Emin, Antigravity]

Subspace \& the zero vector.

$$
\begin{aligned}
& (1,0,0)=(t, E, s) \text { is Perpendicular to } \\
& (0,-1,0)=(t, E, s)
\end{aligned}
$$

This point lies on the y axis on the E-t golden mean parabola.

$$
\begin{gathered}
T=1 / \text { freq. }=1 /(1 / \pi)=\pi \\
s=|E| t \mid \sin \theta \\
E=t=1 \\
s=\sin \theta \\
\theta=\sin 60^{\circ} \\
=\sqrt{3} / 2 \\
=0.866
\end{gathered}
$$

$(3,4,0)$ Perpendicular to $(4,3,0)(-\mathrm{x}, \mathrm{y}, \mathrm{z})$ Perpendicular to $(\mathrm{y}, \mathrm{x}, \mathrm{z})$

$$
\begin{gathered}
-t=E \\
y=x \quad E=t \\
z=z \\
L_{1}=\sqrt{\left(x^{2}+y^{2}+z^{2}\right)}=L_{2}=\sqrt{\left(-x^{2}+y^{2}+z^{2}\right)} \\
(-t, E, s)=(E, t, s)
\end{gathered}
$$

(Likewise, for vectors in the III And IV Quadrant.

Multiple be Operator Matrix

$$
\left[\cos 60^{\circ}-\sin 60^{\circ}, \sin 60^{\circ} \cos 60^{\circ}\right]
$$




$$
\begin{aligned}
& {\left[\begin{array}{llll}
1 / 2 & \sqrt{3} / 2, & \sqrt{3} / 2 & 1 / 2
\end{array}\right]} \\
& |D|=[1 / 2 \times 1 / 2-\sqrt{3} / 2 \sqrt{3} / 2] \\
& \text { [1/4- 3/4] } \\
& \text { Multiple by }[T, S] \\
& 1 / 8+1, \quad 3 / 8+1 \\
& \left.=E_{\text {min }}+1, \quad S . F .+1\right]
\end{aligned}
$$

Period T $=251$

$$
\begin{gathered}
{[2492 / 251,1330 / 251]} \\
{[T, s]} \\
{[\pi, \sin \pi]} \\
{[\pi, 0]}
\end{gathered}
$$

Subspace on te zero vector.

$$
\begin{aligned}
& (1,0,0)=(t, E, s) \quad \text { is Perpendicular to } \\
& (0,-1,0)=(t, E, s)
\end{aligned}
$$

This point lies on the $\mathrm{y}$ axis on the E-t golden mean parabola.

$$
\begin{gathered}
T=1 / \text { freq. }=1 /(1 / \pi)=\pi \\
s=|E| t \mid \sin \theta \\
E=t=1 \\
s=\sin \theta \\
=\sin 60^{\circ} \\
=\sqrt{3} / 2 \\
=0.866
\end{gathered}
$$

$(3,4,0)$ Perpendicular to $(4,3,0)$

$(-\mathrm{x}, \mathrm{y}, \mathrm{z})$ Perpendicular to $(\mathrm{y}, \mathrm{x}, \mathrm{z})$

$$
\begin{gathered}
-t=E \\
y=x \quad E=t \\
z=z \\
L_{1}=\sqrt{\left(x^{2}+y^{2}+z^{2}\right)}=L_{2}=\sqrt{\left(-x^{2}+y^{2}+z^{2}\right)} \\
(-t, E, s)=(E, t, s)
\end{gathered}
$$

(Likewise, for vectors in the III and IV Quadrant.

Because you can't have negative K.E., there is no such thing as negative time, so there is no orthogonal vector to the zero vectors(Figure 2).

Figure 2 Orthogonal vectors.

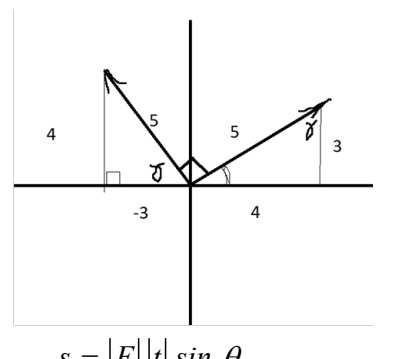

$$
\begin{gathered}
\text { Let } s=z=0 \\
\sin \theta=0 \\
\theta=\pi \\
E=-t \\
E=t \\
s=s=0 \\
E=1 / t \\
1=-t^{2} \\
t=\sqrt{(-1)}=i \\
E=t \\
E=1 / t \\
E{ }^{2}=1 \\
E= \pm 1 \\
\mathbb{R} \varepsilon \mathbb{C} \varepsilon F=0 \\
x+i y \\
y=0 \\
x \varepsilon R \\
x+i y=0 \\
E+t \times t=0 \\
E=t^{2} \\
-E=t^{2} \\
E+t+t^{2}=2=d M / d t \\
1+(-1)=0
\end{gathered}
$$

$0=0$ True

Since time is K.E., and K.E is time, we measure K.E. relative to something - the zero vector. We also know that Mass is the dot product of $E$ and $t$ (Figure 3).

\section{Figure 3 The zero vector.}

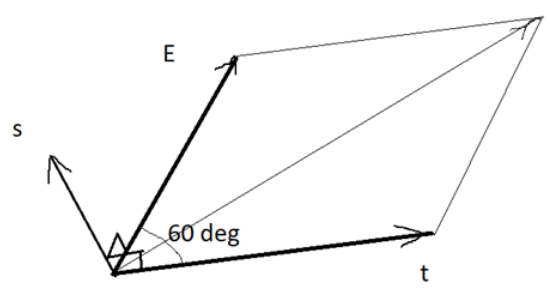

$\{0\}$

$$
\begin{aligned}
M & =|E||t| \cos \theta \\
M & =(1)(1) \cos \theta
\end{aligned}
$$




$$
\theta=1 \mathrm{rad}
$$

And we know that Momentum, $\mathrm{P}$, or $v=\cos \theta$

$$
\begin{aligned}
& \text { So K.E. }=1 / 2 M v^{2} \\
& \begin{aligned}
=1 / 2 & (\cos \theta)(\cos \theta)^{2} \\
= & (\cos \theta)^{3} / 2 \\
= & 0.785 \\
= & 1 / 127.3 \\
= & 1 / \rho \\
\rho & =\text { Density }
\end{aligned}
\end{aligned}
$$

This disproves Einstein's Relativity. There is a stationary point in the universe. It is the Zero Vector. Space and time are absolute. ${ }^{2}$

So this is how the universe crystallized into existence. ${ }^{3}$

$$
M=|E||t| \cos \theta
$$

$$
\begin{gathered}
\text { But } E=P . E .=d M / d t=2 \\
\begin{array}{c}
M=(d M / d t)(1) \cos \theta \\
s=|E| t \mid \sin \theta \\
s=(d M / d t)(1) \sin \theta
\end{array}
\end{gathered}
$$

Density $\rho=M / \mathrm{Vol}$.

$=[d M / d t \cos \theta] /[d M / d t \sin \theta]$

$$
\begin{aligned}
& =\cos \theta / \sin \theta \\
& =\cot \theta
\end{aligned}
$$

$\cot \pi / 3=1 / \sqrt{3}=0.5774=1.0076 \mathrm{rads}=$ Mass $\mathrm{H}^{+}$

Equation of a plane

$$
\begin{gathered}
(r \cdot a) \cdot n=0 \\
(r \cdot a) \cdot(b-a) \times(c \cdot a)=0 \\
(r \cdot a) \cdot n=0 \rightarrow\{r-(0,0,0)\} \cdot(15,0,0)=\mathbb{Z} \\
|E||t| \cos \theta=0 \\
a=(0,0,0)=\mathbb{Z} \text { position vector } \\
(b-a)=(3,0,0)-(0,0, \pi)=(3,0,-\pi) \\
(C-a)=(3,0,0)-(-2,0,0)=(5,0,0) \\
n=(3,0,-\pi) \times(5,0,0)=(15,0,0) \text { Mass Gap } \\
=1 / G=3 / 2 \\
n=c / \quad(d M / d t)=3 / 2=1 / G \\
(r-a) \cdot n=0 \\
{[r-(0,0,0)] \cdot[(3,0,0)]} \\
=|r||0| \cos \theta \\
=0 \\
r \varepsilon R \\
{[r-(0,0,0)] \cdot[(15,0,0)]=0} \\
|r||15| \cos \theta=0
\end{gathered}
$$

$$
\begin{aligned}
& r=0 \text { or } \cos \theta=0 \\
& \theta=\pi / 2,3 \pi / 2
\end{aligned}
$$

$r$ is orthogonal to $n$

$$
\begin{aligned}
& s=|E||t| \sin \theta \\
& 0=0(0)(0) \sin \theta \\
& \theta= \pm \pi ; \pm 2 \pi \\
& E=0=t=0 \\
& M=|E||t| \cos \theta \\
& \mathrm{v} \quad M=\cos \pi=-1 \\
& G_{0}=t^{2} \\
& =0^{2}=0 \\
& E=t=s=G_{0}=0 \\
& M=-1 \\
& \rho=M / \text { Vol. }=-1(\chi) / 19905=-5.02 \sim-4 \pi \\
& 19905(-4 \pi)=\chi \\
& \chi=250.1=\text { Period } T
\end{aligned}
$$

\section{Cusack's gravity equation}

$$
\begin{gathered}
G_{0}=\rho c \sqrt{c} \\
G_{0}=\rho c^{1.5} \\
G_{0}=\rho c^{M A S S G A P}
\end{gathered}
$$$$
=6.522
$$$$
=4 \pi \times 3 \times \sqrt{3}
$$$$
\mid\left[\begin{array}{cccc}
4 & 0 & 0 & 0 \\
0 & 3 & 0 & 0 \\
0 & 0 & \sqrt{3} & 0 \\
0 & 0 & o & -\pi
\end{array}||=G_{0}\right.
$$$$
[4,3, \sqrt{3},-\pi]\left[\begin{array}{c}
1 \\
1 \\
1 \\
-1
\end{array}\right]=M
$$$$
[|D| C \sqrt{C} \Omega]\left[\begin{array}{c}
E \\
t \\
s \\
M
\end{array}\right]=M
$$

Figure 4

Add two vectors

$$
\begin{aligned}
& {\left[\begin{array}{llll}
4 & 3 & \sqrt{3} & -\pi
\end{array}\right]+\left[\begin{array}{llll}
1 & 1 & 1 & -1
\end{array}\right]} \\
& \begin{aligned}
& =\left\{\begin{array}{llll}
5 & 4 & 2.73 & -4.14
\end{array}\right] \\
\text { length } & =\sqrt{\left[\left(\left(5^{2}\right)+\left(4^{2}\right)\right)+\left(2.73^{2}\right)+\left(-4.14^{2}\right)\right]}
\end{aligned} \\
& =781.8=\pi / 4=45^{\circ} \\
& =1 / \rho
\end{aligned}
$$




$$
\begin{gathered}
1 / \rho=\text { Vol. } / \text { Mass }=[d \text { Vol. } / d t] /[d M / d t] \\
=19905 / \sqrt{3} / 2 \\
=57.46=1 \mathrm{rad}
\end{gathered}
$$

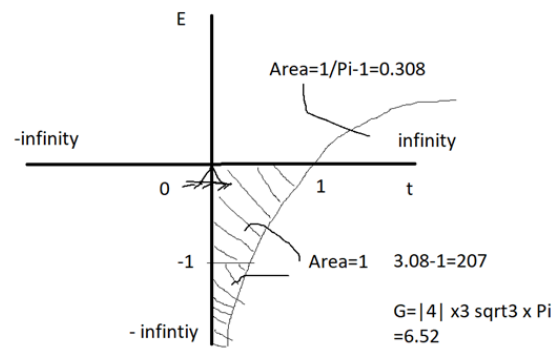

Figure 4 Mass an the Ln function.

Universal vector

$$
\begin{aligned}
& {\left[\begin{array}{llll}
4 & 3 & \sqrt{3} & -\pi
\end{array}\right]=\cdot 615} \\
& {\left[\begin{array}{llll}
1 & 1 & 1 & -1
\end{array}\right]=6}
\end{aligned}
$$

For the angle of this universal vector

$$
\begin{gathered}
c^{2}=a^{2}+b^{2}-2 a b \cos \theta \\
c^{2}=73869^{2}=6^{2}+0.615^{2}-2(6)(0.615) \cos \theta \\
\cos \theta=0.7393 \\
\theta=7387=1 / 0.4233 \\
=1 / \mathrm{cuz} \\
=1 /(\pi-e)
\end{gathered}
$$

So the Universal Vector produces $G_{0} ; \rho, \mathrm{t}=\mathrm{E}=1$ rad;;cuz (or PTEC)(Figure 5).

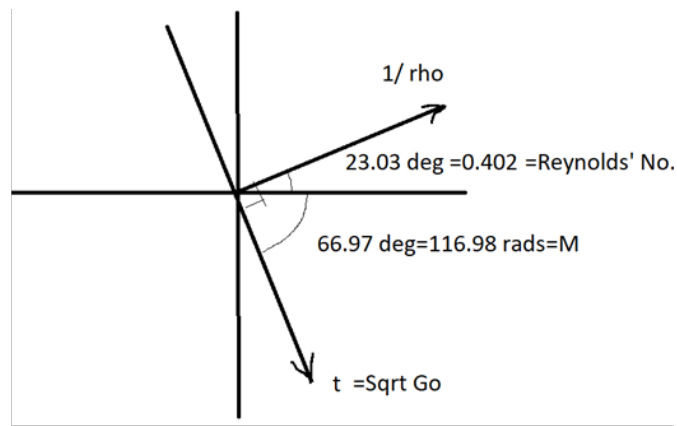

Figure 5 The universal vector and time.
The Norm

$$
\|v\|=\sqrt{(u, v)}
$$

For the universal vector

$$
\begin{gathered}
=\sqrt{(4,3, \sqrt{3}, \pi)} \\
=\sqrt{G_{0}}
\end{gathered}
$$

But we know $G=t^{2}$

$$
=t
$$

And,

$$
\begin{gathered}
\| \lambda t||=|\lambda||||t| \mid \\
\lambda=\text { Eigen value }=3 \\
1 \mathrm{rad}=57.29^{\circ} \\
3 t=3\left(57.29^{\circ}\right) \\
=\sqrt{3}=\text { Eigen vector }
\end{gathered}
$$

Characteristic Equation

$$
x^{2}-x-1=0
$$

Let $x=\pi=$ Operator

$$
\pi^{2}-\pi-1=57.29^{\circ}=1 \mathrm{rad}
$$

\section{Conclusion}

We see that the universe can be well modelled by linear algebra.

\section{Acknowledgements}

None.

\section{Conflict of interest}

The author declares that there is no conflict of interest.

\section{References}

1. Axler S. Linear Algebra Done Right. $3^{\text {rd }}$ ed. Springer, USA; 2015

2. Shilov G. Linear Algebra. USA: Dover; 1971.

3. Cusack P. Astrotheology, Cusack's universe. J of Phys Math. 2016;7(2):8 